\title{
Evolutionary interactions between Drosophila melanogaster and its parasitoid Asobara tabida
}

\author{
D. M. GREEN, A. R. KRAAIJEVELD* \& H. C. J. GODFRAY \\ NERC Centre for Population Biology \& Department of Biology, Imperial College at Silwood Park, Ascot, \\ Berkshire SL5 7PY, U.K.
}

\begin{abstract}
Drosophila melanogaster is attacked by parasitoids that develop internally in the larva. They can defend themselves by a cellular immune response (host resistance), although this can be disabled by parasitoid countermeasures (parasitoid virulence). D. melanogaster and its parasitoids are an excellent system in which to study coevolution experimentally. We designed an experiment to compare changes in resistance and virulence in replicate populations of flies and parasitoids maintained together for approximately 10 fly (five parasitoid) generations. The experiment had three treatments each with three replicates: (A) no parasitoids (B) outbred parasitoids (C) partially inbred parasitoids. Host resistance increased in treatments $\mathrm{B}$ and $\mathrm{C}$ but there was no difference between these treatments. Parasitoid virulence appeared not to change during the experiment. Host larvae in treatments $\mathrm{B}$ and $\mathrm{C}$ fed at lower rates than those in A, evidence of a trade-off between resistance and larval competitive ability. We found no evidence for local adaptation, as hosts from the different replicates of treatment $\mathrm{C}$ performed no differently against parasitoids from the same and other replicates. Also, we found no evidence for the evolution of behavioural traits in the host that could lead to lower probabilities of being attacked. Comparing the evolution of host resistance in these seminatural settings with that in artificial selection experiments provides insight into how the conflicting selection pressures on host resistance interact.
\end{abstract}

Keywords: coevolution, Drosophila, encapsulation, parasitoid, resistance, virulence.

\section{Introduction}

Predators, parasites and pathogens are likely to exert strong and reciprocal selection pressures on their hosts and prey. The course of such coevolution depends on many factors, including the extent of genetic variation for traits influencing attack and defence, trade-offs between these traits and other components of fitness, and the specificity of the interaction between different genotypes in the natural enemy and in its prey or host.

An attractive model system for investigating these questions is Drosophila and its parasitoid wasps. Drosophila larvae are attacked in the wild by a number of parasitoids in the families Braconidae and Eucoilidae (Hymenoptera). The female wasp oviposits into the fly larva and its progeny feeds internally, eventually killing the host at the pupal stage. The host may be able to defend itself by mounting a cellular immune response against the parasitoid egg: cells circulating in the

*Correspondence. E-mail: a.kraayeveld@ic.ac.uk haemocoel recognize the parasitoid egg as foreign and cause other cells to aggregate around the egg. The cells melanize and form a capsule that results in the death of the parasitoid, either by asphyxiation or through the release of toxic compounds (Salt, 1970; Nappi, 1981; Rizki \& Rizki, 1984; Nappi \& Vass, 1993; Nappi et al., 1995). Different species of parasitoids have evolved a variety of adaptations to counter encapsulation, including molecular mimicry of the host and the active destruction of host immune cells (Beckage et al., 1993; Strand \& Pech, 1995). In the context of host-parasitoid interactions, host resistance is defined as the probability of killing the parasitoid egg or larva, and parasitoid virulence as the probability of overcoming host defences. These terms are thus employed in a slightly different way from their usage in traditional parasitology.

Genetic variation in resistance appears to be widespread in Drosophila melanogaster. This has been shown by many isofemale line studies by Carton and coworkers (reviewed in Carton \& Nappi, 1991 and Kraaijeveld et al., 1998) and in addition, we and other groups have 
shown that Drosophila melanogaster can be artificially selected for increased resistance to its larval parasitoids. For example, Hughes \& Sokolowski (1996) maintained three replicate populations of $D$. melanogaster with the braconid Asobara tabida for 19 generations, and compared their resistance levels with three control populations cultured without parasitoids. Larvae from experimental lines had a resistance level of $39 \%$ compared with control larvae of $23 \%$. The risk of parasitism in these experiments was quite low (6-8\% on average). Using much more intense selection pressure (breeding only from flies that had successfully encapsulated parasitoid eggs), Kraaijeveld \& Godfray (1997) increased D. melanogaster resistance to A. tabida from $5 \%$ to $60 \%$ in five generations, while Fellowes et al. (1998) were able to raise the resistance of D. melanogaster to the eucoilid Leptopilina boulardi from $0.5 \%$ to $45 \%$ over the same period of time (in both cases, the percentages are the means of four replicate lines). Kraaijeveld \& Godfray (1997) and Fellowes et al. (1998) found a trade-off between increased resistance and larval competitive ability, though the selected flies only suffered reduced fitness at moderate to high intensities of resource competition. A possible proximate explanation for the reduced competitive ability is the observation that selected larvae feed at a slower rate than control larvae (Fellowes et al., 1999).

In additional to physiological defences, parasitoid attack can also select for the evolution of behavioural avoidance mechanisms (Hochberg, 1997). A polymorphism in larval foraging behaviour exists in field populations of $D$. melanogaster. Larva with the 'rover' phenotype move around much more on a patch of food than 'sitter' larvae (Sokolowski, 1980; Sokolowski et al., 1986; Osborne et al., 1997). This behavioural polymorphism has a very simple genetic basis: a one-locus, twoallele system, with the rover allele dominant over the sitter allele (De Belle \& Sokolowksi, 1987). Rover and sitter larvae differ in the degree to which they are discovered by parasitoids (which frequently rely on host movement for detection) and it has been suggested that the risk of attack will influence the frequency of the two alleles (Carton \& Sokolowski, 1992; Kraaijeveld \& van Alphen, 1995a). However, in the experiment described above, Hughes \& Sokolowski (1996) found no difference in the proportions of rovers and sitters in control populations and those exposed to parasitism.

Evidence for genetic variation in virulence amongst Drosophila parasitoids is more equivocal than for resistance in the host. A limited number of isofemale line studies have shown some genetic variation (Carton et al., 1989; Dupas et al., 1998), but whether or not this is additive has not been established; there have been no replicated selection experiments. However, outside
Drosophila parasitoids, Henter (1995) found substantial additive genetic variation in the virulence of an aphid parasitoid, Aphidius ervi. A few studies have investigated whether there has been local adaptation between host and parasitoid genotypes (Carton, 1984; Carton \& Nappi, 1991), the evidence currently being at best very weak (Kraaijeveld et al., 1998).

In the experiments mentioned above that selected for host resistance, changes in virulence in the parasitoid were prevented. Here we describe replicated experiments in which large populations of D. melanogaster and its larval parasitoid Asobara tabida are allowed to coevolve in a more natural setting. The aim of the work reported here was to test four hypotheses:

1 When large outbred populations of hosts and parasitoids are maintained together in a seminatural setting, are there changes in either or both levels of resistance and virulence, and how do changes in resistance and correlated traits (larval feeding rates) compare to the results obtained from artificial selection experiments?

2 Does reducing the genetic variation of one partner (in our case by partially inbreeding the parasitoid) influence the outcome of the interaction?

3 Is there evidence for local adaptation - that is, do hosts/parasitoids perform better against parasitoids/ hosts from the population with which they evolved than against other populations?

4 Is there evidence for the evolution of avoidance behaviour, i.e. is there a change in the proportion of rovers and sitters in the populations exposed to parasitoids compared to the control populations?

\section{Methods}

\section{Insects}

The D. melanogaster used in this study originated from a large $(>250)$ collection of flies from Leiden, the Netherlands. The strain had been maintained as a large outbred population with ad libitum larval food in the laboratory for two years prior to the experiment, and is the same as that used by Kraaijeveld \& Godfray (1997) and Fellowes et al. (1998). The A. tabida used in the experiment came from a culture founded from over 100 female wasps collected at Silwood Park, southern England, a year before the start of the experiment. The wasps had been collected and maintained in a way designed to minimize the loss of natural genetic variability (five separate outbred populations, each based on 50 females each generation). Prior to the experiment, they had been cultured using D. subobscura as a host. This species has never been recorded to encapsulate $A$. tabida eggs (Kraaijeveld \& van der Wel, 
1994) and, hence, the wasp is unlikely to have been subject to selection for increased virulence before the start of the experiment. In assaying for host resistance we used a laboratory strain of $A$. tabida originally collected from Sospel in southern France. Stock cultures of all host and parasitoid strains were maintained in $150 \mathrm{~mL}$ bottles with yeast/sugar medium and live baker's yeast at $20^{\circ} \mathrm{C}$.

There is considerable geographical variation in resistance and virulence in D. melanogaster and A. tabida (Kraaijeveld \& van Alphen, 1994, 1995b; Kraaijeveld \& Godfray, 1999). The northern strains of host and parasitoid used in the experiments have relatively low values of resistance and virulence. The Sospel $A$. tabida strain used in the resistance assays has a much higher virulence than the experimental strain (Kraaijeveld \& van Alphen, 1994).

\section{Experimental design}

The experiment consisted of three treatments, each replicated three times. The treatments were: (A) fly populations maintained without parasitoids; (B) fly populations maintained with outbred parasitoids; and (C) fly populations maintained with partially inbred parasitoids. The parasitoid lines for the three replicates of treatment $\mathrm{C}$ were each derived from a single sib-mated female; a different female was used for each of the three lines. All populations were kept in a $20^{\circ} \mathrm{C}$ constant temperature room with a 16:8 light:dark regime.

Each replicate consisted of three muslin-covered wireframe cages: one containing adult flies, one containing adult wasps (both measuring $30 \times 60 \times 30 \mathrm{~cm}$; the wasp cage was empty in treatment A), and a rearing cage (measuring $75 \times 60 \times 75 \mathrm{~cm}$ ). All cages were provided with fresh water and cages with wasps also contained some honey. Twice a week a tray $(10 \times 10 \times 5 \mathrm{~cm})$ containing $16 \mathrm{~g}$ of water-saturated vermiculite and four discs of banana (combined weight: $65 \mathrm{~g}$ ) was placed for $24 \mathrm{~h}$ in the adult fly cage where oviposition occurred. The banana discs (with the skin intact) had been soaked in yeast suspension ( $25 \mathrm{~g}$ of yeast in $100 \mathrm{~mL}$ water) prior to use. The trays were then placed for one week in the parasitoid cage for parasitism to take place, after which they were transferred to the rearing cage where the flies and parasitoids completed development. Once a week about 100 adult parasitoids and up to 500 adult flies (on some occasions fewer parasitoids or flies were available) were transferred from the rearing cages to the adult fly and parasitoid cages, respectively; trays were discarded 6 weeks after fly oviposition. We chose this experimental design rather than keeping all host and parasitoid stages together in one cage per replicate because preliminary experiments with the latter set-up had found substantial interference of parasitoid oviposition by flies crowding on the banana discs. Experiments were initiated with 120 flies and 100 wasps with an equal ratio sex ratio and were run for five months which is approximately $10 \mathrm{fly}$ generations and 5 parasitoid generations.

\section{Resistance and virulence assays}

The resistance and virulence assays are the same as those of Kraaijeveld \& van Alphen (1994, 1995b). For each assay, five $5 \mathrm{~cm}$ Petri dishes were prepared with a thin layer of agar, and a patch of yeast suspension (of roughly $1 \mathrm{~cm}$ diameter). Twenty second-instar larvae were placed in each yeast patch and two female parasitoids introduced to each dish. Dishes were kept in a $20^{\circ} \mathrm{C}$ constant temperature room for two hours, after which the wasps were removed. The host larvae were reared in the dishes at $20^{\circ} \pm 0.5^{\circ} \mathrm{C}$ for five days after which all hosts were dissected and the number with encapsulated and nonencapsulated parasitoids counted. About $10 \%$ of hosts contain more than one parasitoid egg but these are not used in the calculation of resistance or virulence.

In comparing resistance and virulence at the beginning and end of the experiment, it is important to challenge the host or parasitoid with a strain that has not itself changed over this period. For resistance, this is not a problem as our standard parasitoid, the Sospel strain of A. tabida, is an inbred laboratory strain whose virulence has remained stable over many years. We were not as confident that our standard Drosophila (the outbred Leiden strain) was equally stable and, hence, we measure the virulence of the parasitoids in the experiment relative to the Sospel strain (with virulence of experimental and standard parasitoids expressed as the arc-sine of the proportion of wasp eggs surviving).

\section{Feeding rate, body size and path length}

In order to measure larval feeding rates, we followed the procedure in Fellowes et al. (1999). Second-instar larvae were placed in a $5-\mathrm{cm}$ Petri dish containing a layer of agar coated with a film of a $25 \%$ yeast suspension. For 15 larvae from each replicate, the time taken for 15 retractions of the cephalopharyngeal skeleton (the mouth-hooks) to occur was recorded.

The size of adult flies in the different treatments was measured as this provides information on the intensity of competition for food amongst the larvae. Towards the end of the experiment, two samples (3 weeks apart) of 15 flies of each sex were taken from each cage and the length of their wings (from the distal end of the costal cell to the wing tip) measured to the nearest $25 \mu \mathrm{m}$. 
Differences in the frequency of rover and sitter larvae can be assessed by measuring the distribution of larval movement rates or path lengths. We followed and slightly adapted the protocol of Sokolowski (1980). Second-instar larvae were allowed to crawl for $5 \mathrm{~min}$ in a $5-\mathrm{cm}$ Petri-dish containing a layer of agar coated with a thin layer of a $33 \%$ yeast suspension. The trail left by the larva was traced onto paper using a camera lucida and then measured with a cartographic distance-measuring tool. From each cage, we measured the path length of 25 larvae. All measurements were made in a constant temperature room, at $20^{\circ} \mathrm{C}$, with constant light intensity and $70 \%$ relative humidity. After measurement, we reared all larvae individually in small vials with ad libitum food and discarded data from individuals that did not successfully develop into adults (which happened in a very small minority of cases).

\section{Statistical analyses}

Resistance and virulence are measured as proportions and hence were analysed using logistic analysis of deviance (using the GLIM statistical package). The data were checked for error variance overdispersion, which was not found. Relative virulence and feeding rate was analysed using standard ANOvA. Differences in wing length between sex, treatment and sampling period were analysed using $t$-tests. The local adaptation assay was analysed using ANOvA of angular transformed data, followed by analysis of the residuals with a $t$-test. Path lengths were analysed by a $t$-test on the mean path length for each cage.

\section{Results}

Parasitoid virulence was measured relative to the inbred Sospel strain of $A$. tabida to account for any change in the resistance of our standard host culture over the course of the experiment. Although relative parasitoid virulence in the experimental populations tended to be higher than in the base population (Fig. 1), we found no significant difference between treatments B \& C, nor between parasitoids at the end of the experiment and in our base population (ANOVA, $F_{2,8}=0.948, P=0.43$ ).

We assayed resistance of flies from the nine replicates against our standard reference strain (Sospel) of A. tabida (Fig. 2). Overall there was a significant effect of treatment $\left(\chi_{2}^{2}=9.50, P=0.0087\right)$. Resistance in flies subject to attack by partially inbred wasps was slightly higher than that in the outbred replicates, but this trend was a long way from significance $\left(\chi_{1}^{2}=1.76, P=0.18\right)$. Pooling treatments $\mathrm{B}$ and $\mathrm{C}$, the average resistance of control flies was $17.5 \%$ and of flies exposed to parasitoids $30.4 \%\left(\chi_{1}^{2}=7.73, P=0.0054\right)$.

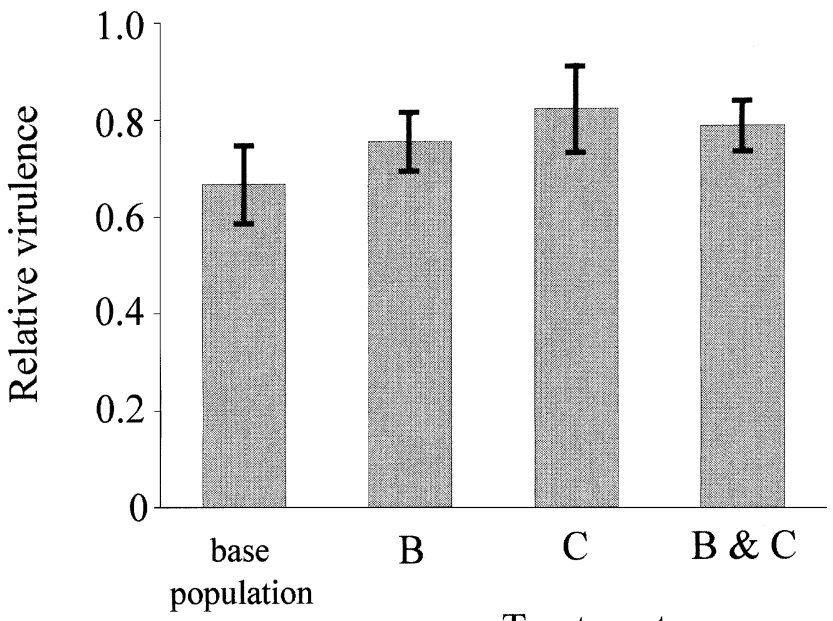

Fig. 1 The virulence of Asobara tabida in the base population and in treatments B \& C measured relative to a standard inbred strain (see text). The pooled data from treatments B \& $\mathrm{C}$ are also shown. Bars represent SE.

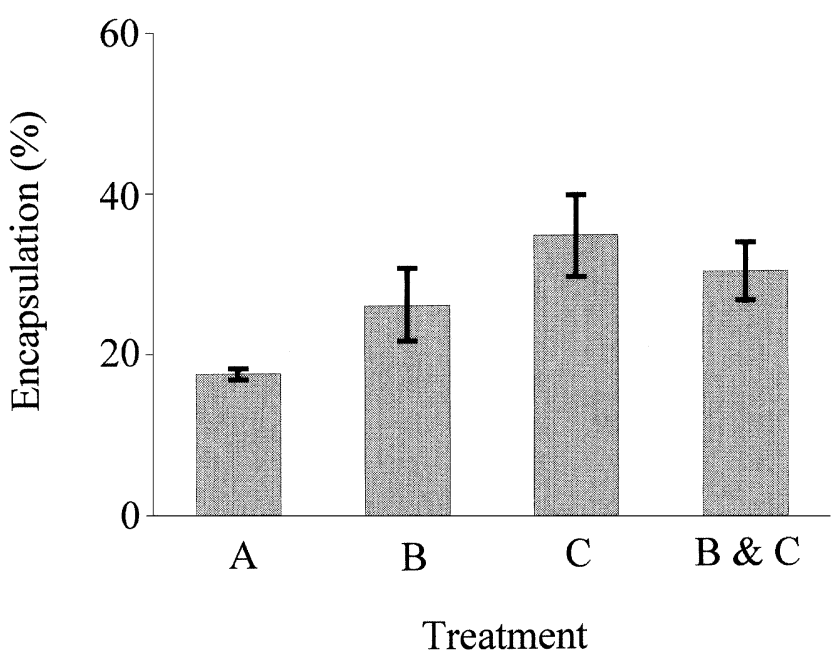

Fig. 2 The percentage of flies successfully encapsulating eggs of a standard strain of wasp in the three treatments (with pooled data from the two parasitoid treatments B \& C also shown). Bars represent SE.

We investigated whether increased resistance was associated with slower rates of feeding, a trait previously found to be correlated with resistance (Fellowes et al., 1999). Feeding rates of fly larvae from the two parasitoid treatments were very similar and were pooled. Overall, fly larvae from the parasitoid treatments fed significantly more slowly than larvae from the control treatment $\left(F_{1,7}=8.08, \quad P=0.025\right.$; Fig. 3).

We wanted to know whether levels of resource competition varied amongst treatments as this might 


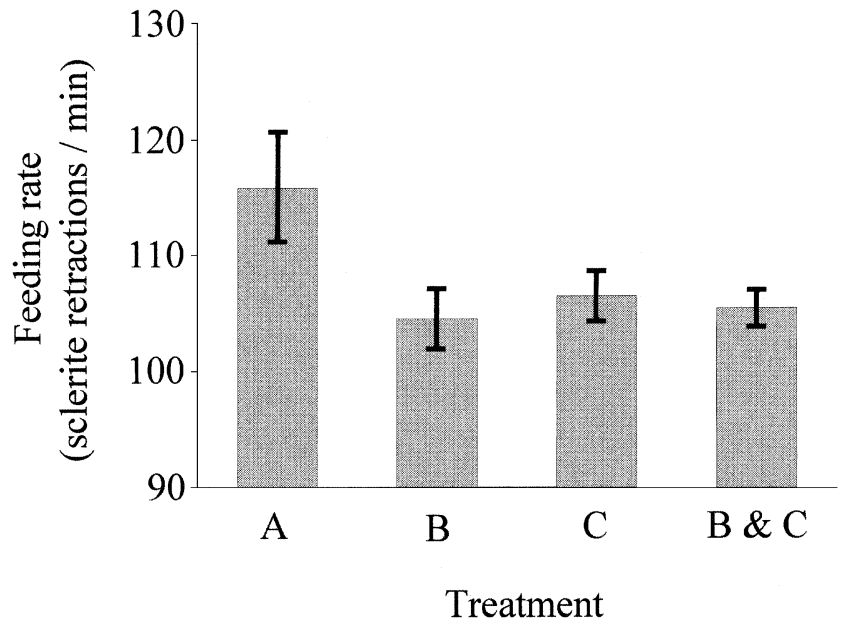

Fig. 3 The feeding rate of flies (measured as the number of retractions per minute of the sclerites in the cephalopharyngeal skeleton) in the three treatments (with pooled data from the two parasitoid treatments B \& C also shown). Bars represent SE.

lead to differences in the selection pressures experienced by the flies. It is difficult to assess directly the intensity of resource competition in this type of experimental set-up. However, because adult size declines with increasing larval competition it can be used as a surrogate measure of competition. Fly sizes (wing lengths) were measured on two occasions but no significant differences were found between dates in either sex (paired $t$-test; males: $t_{8}=1.320, P=0.224$; females: $t_{8}=0.322, P=0.755$ ); thus the two samples were pooled. As expected, there was a significant difference between the sexes ( $t$-test; $t_{16}=3.250, \quad P=0.005$ ), but no significant difference amongst flies from the three treatments $(t$-test; males: $t_{7}=1.070, P=0.319$; females: $\left.t_{7}=0.765, P=0.470\right)$. The overall mean wing length for males and females was $1.43 \mathrm{~mm}$ and $1.58 \mathrm{~mm}$, respectively. This size corresponds to levels of competition at which Kraaijeveld \& Godfray (1997) and Fellowes et al. (1998) found that flies selected for increased resistance manifested reduced competitive ability.

To test for local adaptation, we assayed the resistance of the three fly populations in treatment $\mathrm{C}$ (partially inbred wasps) against the three parasitoid lines in that treatment (upper panel of Fig. 4). If local adaptation had occurred, we might have expected different results when hosts were paired with the parasitoid line with which they had evolved, compared to when they were paired with one of the two other parasitoid lines. To test this, we first fitted an ANOvA model to the data, with wasp line and fly line as factors. As expected, this model accounted for a large proportion $\left(r^{2}=98.9 \%\right)$ of the variance, much of which could be explained by the third parasitoid population from treatment $\mathrm{C}$, which had consistently low virulence. We then analysed the residuals of this model (lower panel of Fig. 4) with a $t$-test (a nonparametric test gave the same results). No difference was found in the magnitude of residuals from 'sympatric' and 'allopatric' combinations $\left(t_{7}=1.270\right.$, $P=0.243$ ). We used a two-tailed test, as we had no $a$ priori expectation about whether local adaptation would result in hosts becoming better adapted to their parasitoids or vice versa.
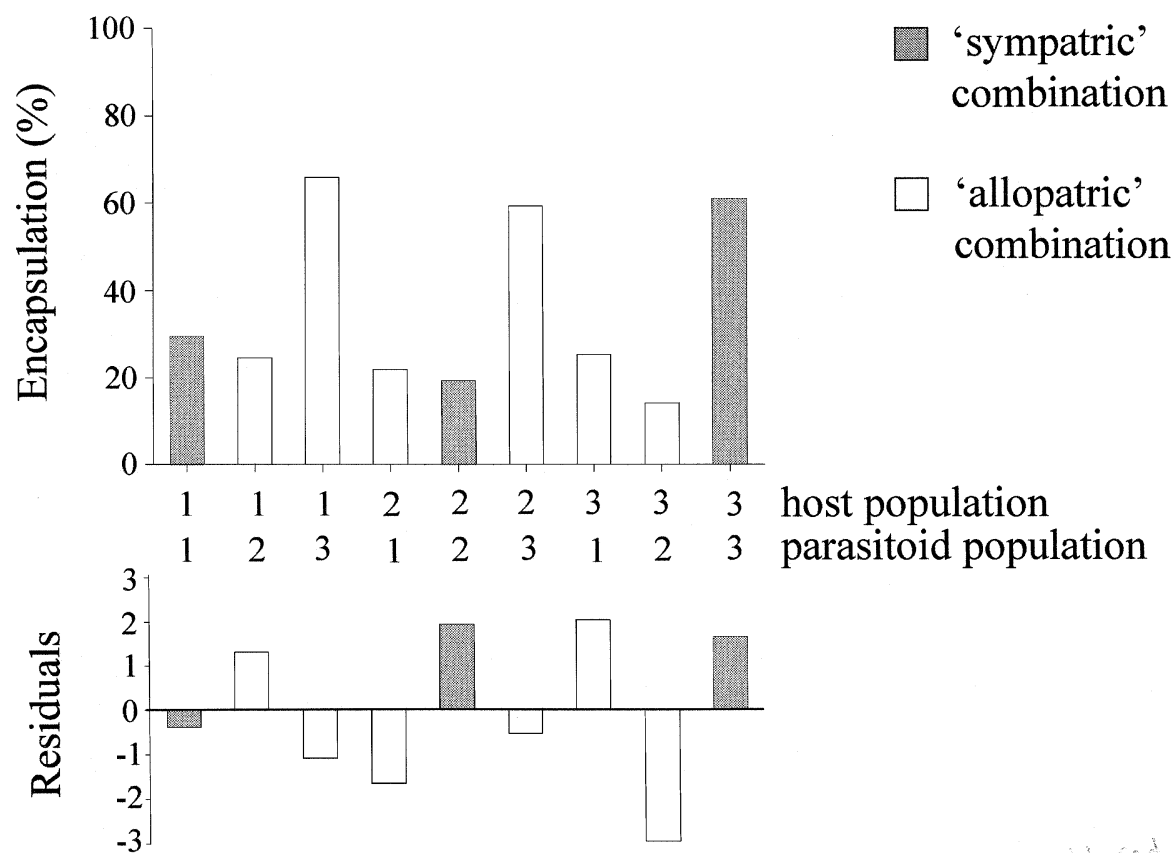

Fig. 4 Upper panel: the percentage of wasp eggs encapsulated when flies from the three replicates of treatment $\mathrm{C}$ (numbered 1, $2 \& 3$ ) were exposed to parasitoids with which they had potentially coevolved ('sympatric' combinations, shaded) and those from the other replicates ('allopatric' combinations, unshaded). Lower panel: the residuals from a fitted model including host and parasitoid populations as main effects. 
The frequency distribution of path lengths (Fig. 5) did not differ between treatments. A $t$-test on the mean value for each cage, again pooling the two parasitoid treatments, showed no difference in path length between larvae from control and parasitoid-exposed populations (overall control mean: $25.9 \mathrm{~mm}$; overall parasitoidexposed mean: $\left.32.7 \mathrm{~mm} ; t_{7}=-0.986, P=0.357\right)$. The large standard errors in treatment $\mathrm{A}$ and the apparent increase in the mean path lengths in the parasitoid cages
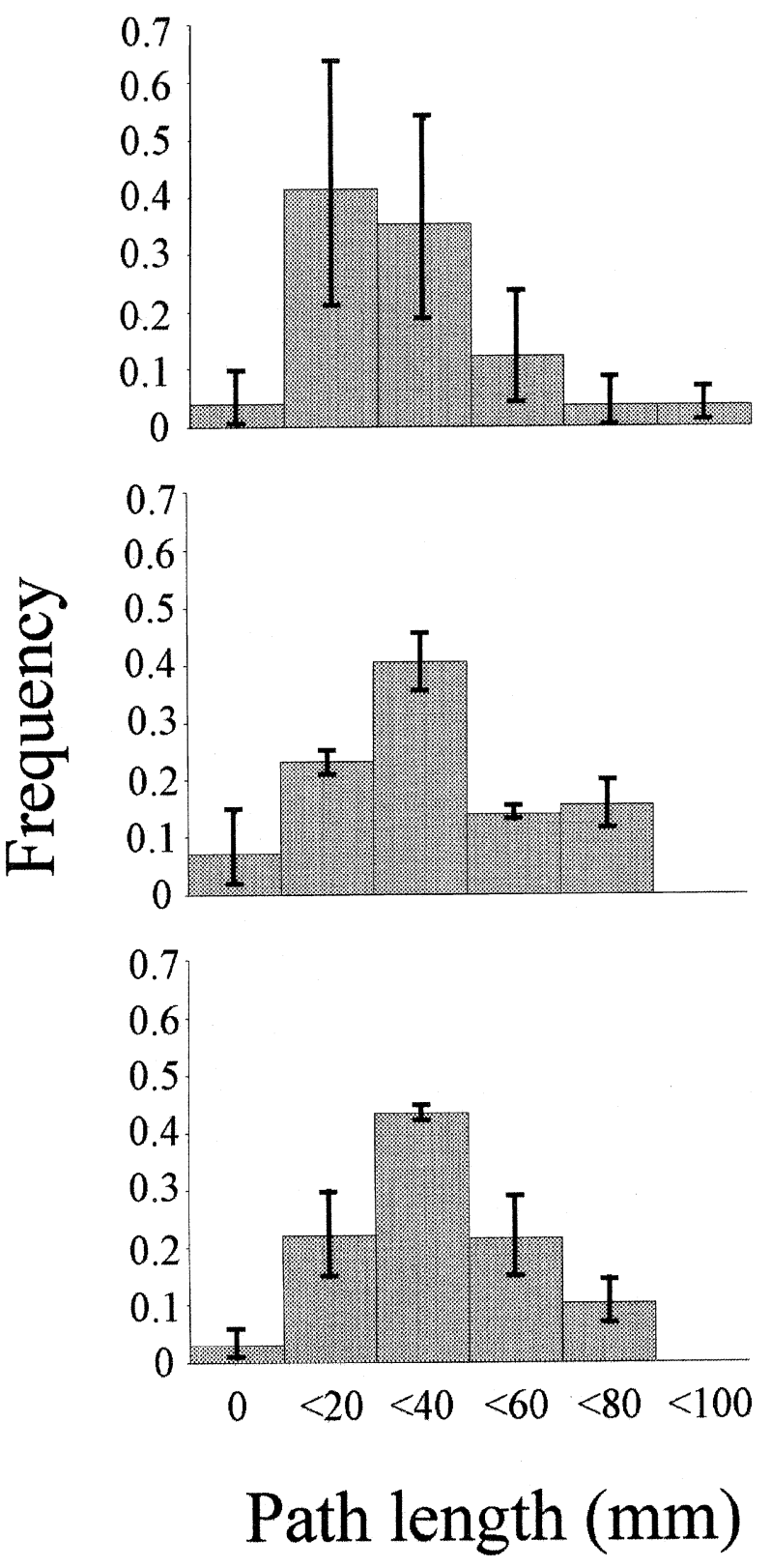

Fig. 5 The distribution of path lengths of fly larvae from the three treatments. Path lengths are categorized as 0 (no movement) and in cumulative length bands of $20 \mathrm{~mm}$. Bars represent $\mathrm{SE}$. are the result of the path lengths from one control cage being virtually all in the range $0-20 \mathrm{~mm}$, whereas the path length distributions from the other two control cages were very similar to those from the two parasitoid treatments (i.e. symmetrical around the $20-40 \mathrm{~mm}$ category). Very few larvae showed a typical 'sitter' phenotype (with path lengths equal or very close to 0 ).

\section{Discussion}

We maintained populations of hosts and parasitoids in circumstances where each could exert significant selection pressure on the other and, hence, where coevolutionary interactions might be expected to occur. We found that $D$. melanogaster populations evolved higher resistance to parasitoids compared to controls. Although there was a trend towards the evolution of higher virulence in A. tabida, it was not significant and therefore we failed to detect coevolution. We also found no evidence of local adaptation within different hostparasitoid interactions.

The response of the host is in line with other studies of D. melanogaster, which have shown that increased rates of parasitism favour higher resistance. Compared with our earlier artificial selection study of the same strain of host (Kraaijeveld \& Godfray, 1997), the response reported here is weaker with resistance rising to approximately half that in the earlier experiment. Part of the reason for this is the difference in the intensity of parasitoid attack: Kraaijeveld \& Godfray (1997) imposed an effective rate of $100 \%$ while we know from the numbers of flies and parasitoids emerging in the population cages that, given the measured rates of resistance and virulence, many hosts are escaping attack. Another factor affecting observed levels of resistance is the reduced competitive ability shown by larvae selected for higher resistance (Kraaijeveld \& Godfray, 1997; Fellowes et al., 1998). Fellowes et al. (1999) argued that the proximate cause of the lower competitive ability was reduced feeding rate, a trait that has long been implicated in density-dependent selection in Drosophila (Joshi \& Mueller, 1988, 1996). The fly populations exposed here to parasitoid attack had significantly lower larval feeding rates, and their body size indicated resource competition to be significant, which implies potentially that they suffer from lower competitive ability. However, competitive ability is likely to be frequency dependent and its worse consequences may not be experienced within a population where all flies are feeding more slowly.

A further potential explanation for the weaker response compared to the artificial selection lines is that our populations exposed to parasitism evolved behavioural traits leading to lower probabilities of being 
attacked in the first place and thus reducing the selection pressure on resistance. But, like Hughes \& Sokolowski (1996), we did not find evidence for a shift in the proportions of rovers and sitters between our control and treatment populations (although the trend was in the direction opposite to that predicted). Note, however, that we used measurements of larval path length as an indicator of this trait and have not shown formally that the rover/sitter polymorphism occurs in our population.

Are there other factors apart from the presence of parasitoids that might lead to differences in resistance between treatments $\mathrm{A}$ and $\mathrm{B} / \mathrm{C}$ ? One possibility is that the intensity of competition is stronger when parasitoids are absent. If competitive ability was selected for in treatment $\mathrm{A}$, then, given the trade-off between competitive ability and resistance, the results might be explained by a reduction in resistance in treatment $\mathrm{A}$ rather than an increase in treatments B and C. Several lines of evidence argue against this occurring in our experiments. First, the population size of flies in replicates of the three treatments was kept approximately constant. However, it is possible that parasitized larvae are weaker competitors than unparasitized larvae so that surviving flies in treatments $\mathrm{B}$ and $\mathrm{C}$ will have experienced reduced competition. Secondly, we found no difference in adult fly size across treatments and, as explained before, adult size can be used as a surrogate measure of larval competition. Finally, the resistance of control flies is similar to that in the base population, which indicates flies in treatments $\mathrm{B}$ and $\mathrm{C}$ have indeed increased their level of resistance. Thus the results do seem to be a direct consequence of selection imposed by parasitoid attack, even though the magnitude of the response may very well have been modulated by the constant background level of larval competition experienced by flies in our population cages.

There are at least three explanations for the nonsignificant increase in parasitoid virulence. (i) lack of statistical power in the experiment; (ii) insufficient selection pressures relative to the length of the experiment; (iii) a lack of genetic variation upon which selection can act. In our experimental design, populations are the unit of replication and the logistic difficulties of maintaining a large number of populations simultaneously limit the number of replicates and hence the statistical power of the experiment. Between-population variability in virulence tended to be higher than that for resistance and thus detecting changes in the former was more difficult. However, results from other experiments suggest that the asymmetric response of resistance and virulence is real. In on-going replicated artificial selection experiments using the same $A$. tabida population, but a different (more resistant) host strain, we could detect a consistent increase in virulence across the five selection lines only after 15 generations (Kraaijeveld and Godfray, unpubl. results), which is much longer than the approximately 5 parasitoid generations of the population cage experiment. Thus, there does appear to be some, but limited, additive genetic variation for virulence in our A. tabida population, although less than the additive genetic variation for resistance in the host (Kraaijeveld \& Godfray, 1997).

Other factors that may influence the relative changes in resistance and virulence include differences in host and parasitoid generation times. Because Drosophila parasitoid generation times are longer than those of their hosts, the cumulative force of selection on the parasitoids in the experiment is less than that on the host. Wajnberg et al. $(1985,1990)$ have shown that under larval crowding the efficiency of the encapsulation response of $D$. melanogaster larvae against a different parasitoid species, Leptopilina boulardi, decreases. The level of competition we observed in the population cages might be sufficient to reduce the intensity of selection on virulence. In our partial inbred treatment, one of the isofemale parasitoid lines (number 3) displayed consistently lower virulence than the others. We do not know the reason for this, but suspect that inbreeding has unmasked a deleterious mutation that in some way compromises the wasp's ability to disable host resistance.

Is the presence of high levels of additive genetic variation in host resistance and lower levels of additive genetic variation in parasitoid virulence likely to be widespread in the field? It is hard to answer this question at the moment because genetic variation in virulence has been studied in so few parasitoids. A few isofemale line studies have revealed genetic variation in virulence (Dupas et al., 1998), and even localized some of the genes involved, but whether the variation is additive or higher order is not yet clear. In contrast, Henter \& Via (1995) and Henter (1995) found considerable genetic variation for both resistance in pea aphid (Acyrthosiphon pisum) and virulence of its parasitoid Aphidius ervi. Interestingly, in a different system, Jaenike \& Dombeck (1998) recently failed to select for changes in virulence in a nematode parasite of Drosophila.

The evolutionary interaction between parasitoids and their hosts is intrinsically asymmetrical, as every individual parasitoid needs a host but not vice versa (Sasaki \& Godfray, 1999). This could mean that virulence is generally under stronger selection pressure than resistance and thus lower levels of genetic variation for virulence than for resistance may be a general pattern in host-parasitoid systems. Given that rapid progress is being made in identifying the genes involved in variation in host resistance (Carton et al., 1992; Orr \& Irving, 
1997; Poirie et al., 2000), we believe that Drosophila and its parasitoids are an excellent model system to investigate these types of evolutionary and coevolutionary interactions from a combined genetic and ecological viewpoint.

\section{Acknowledgements}

DMG was supported by a NERC studentship; we thank Mark Fellowes and two anonymous referees for helpful comments.

\section{References}

BECKAGE, N. E., THOMPSON, S. N. AND FEDERICI, B. A. 1993. Parasites and Pathogens of Insects, Vol. 1: Parasites. Academic Press, San Diego, CA.

CARTON, Y. 1984. Analyse expérimentale de trois niveaux d'interaction entre Drosophila melanogaster et le parasite Leptopilina boulardi (sympatrie, allopatrie, xénopatrie). Génét. Sél. Evol., 16, 417-430.

CARTON, Y., CAPY, P. AND NAPPI, A. J. 1989. Genetic variability of host-parasite relationship traits: utilization of isofemale lines in a Drosophila simulans parasitic wasp. Génét. Sél. Evol., 21, 437-446.

CARTON, Y., FREY, F. AND NAPPI, A. J. 1992. Genetic determinism of the cellular immune reaction in Drosophila melanogaster. Heredity, 69, 393-399.

CARTON, Y. AND NAPPI, A. J. 1991. The Drosophila immune reaction and the parasitoid capacity to evade it: genetic and coevolutionary aspects. Acta Oecologica, 12, 89-104.

CARTON, Y. AND SOKOLOWSKI, M. B. 1992. Interactions between searching strategies of Drosophila parasitoids and the polymorphic behavior of their hosts. J. Insect Behav., 5, 161-175.

DE BELLE, J. S. AND SOKOLOWKSI, M. B. 1987. Heredity of rover/ sitter: alternative foraging strategies of Drosophila melanogaster larvae. Heredity, 59, 73-83.

DUPAS, S., FREY, F. AND CARTON, Y. 1998. A single parasitoid segregating factor controls immune suppression in Drosophila. J. Hered., 89, 306-311.

FELlOWES, M. D. E., KRAAIJEVELD, A. R. AND GODFRAY, H. C. J. 1998. Trade-off associated with selection for increased ability to resist parasitoid attack in Drosophila melanogaster. Proc. R. Soc. B, 265, 1553-1558.

FELloweS, M. D. E., KRAAIJEVELD, A. R. AND GODFRAY, H. C. J. 1999. Association between feeding rate and defence against parasitoids in Drosophila melanogaster. Evolution, 53, 1302-1305.

HENTER, H. J. 1995. The potential for coevolution in a hostparasitoid system. 2. Genetic variation within a population of wasps in the ability to parasitize an aphid host. Evolution, 49, 439-445.

HENTER, H. J. AND VIA, S. 1995. The potential for coevolution in a host-parasitoid system. 1. Genetic variation within an aphid population in susceptibility to a parasitic wasp. Evolution, 49, 427-438.
HOCHBERG, M. E. 1997. Hide or fight? The competitive evolution of concealment and encapsulation in parasitoid-host associations. Oikos, 80, 342-352.

HUGHES, K. AND SOKOLOWSKI, M. B. 1996. Natural selection in the laboratory for a change in resistance by Drosophila melanogaster to the parasitoid wasp Asobara tabida. J. Insect Behav., 9, 477-491.

JAENIKE, J. AND DOMBECK, I. 1998. General-purpose genotypes for host species utilization in a nematode parasite of Drosophila. Evolution, 52, 832-840.

JOSHI, A. AND MUELLER, L. D. 1988. Evolution of higher feeding rate in Drosophila due to density dependent natural selection. Evolution, 42, 1090-1093.

JOSHI, A. AND MUELLER, L. D. 1996. Density-dependent natural selection in Drosophila: trade-offs between larval food acquisition and utilization. Evol. Ecol., 10, 463-474.

KRAAIJEVELD, A. R. AND VAN ALPHEN, J. J. M. 1994. Geographical variation in resistance of the parasitoid Asobara tabida against encapsulation by Drosophila melanogaster larvae: the mechanism explored. Physiol. Ent., 19, 9-14.

KRAAIJEVELD, A. R. AND VAN ALPHEN, J. J. M. 1995a. Foraging behavior and encapsulation ability of Drosophila melanogaster larvae: correlated polymorphisms? (Diptera: Drosophilidae). J. Insect Behav., 8, 305-314.

KRAAIJEVELD, A. R. AND VAN ALPHEN, J. J. M. 1995b. Geographical variation in encapsulation ability of Drosophila melanogaster larvae and evidence for parasitoid-specific components. Evol. Ecol., 9, 10-17.

KRAAIJEVELD, A. R., VAN ALPHEN, J. J. M. AND GODFRAY, H. C. J. 1998. The coevolution of host resistance and parasitoid virulence. Parasitology, 116, S29-S45.

KRAAIJEVELD, A. R. AND GODFRAY, H. C. J. 1997. Trade-off between parasitoid resistance and larval competitive ability in Drosophila melanogaster. Nature, 389, 278-280.

KRAAIJEVELD, A. R. AND GODFRAY, H. C. J. 1999. Geographic patterns in the evolution of resistance and virulence in Drosophila and its parasitoids. Am. Nat., 153, S61-S74.

KRAAIJEVELD, A. R. AND VAN DER WEL, N. N. 1994. Geographical variation in reproductive success of the parasitoid Asobara tabida in larvae of several Drosophila species. Ecol. Entomol., 19, 221-229.

NAPPI, A. J. 1981. Cellular immune response of Drosophila melanogaster against Asobara tabida. Parasitology, 83, 319-324.

NAPPI, A. J. AND VASS, E. 1993. Melanogenesis and the generation of cytotoxic molecules during insect cellular immune reactions. Pigment Cell Res., 6, 117-126.

NAPPI, A. J., VASS, E., FREY, F. AND CARTON, Y. . 1995. Superoxide anion generation in Drosophila during melanotic encapsulation of parasites. Eur. J. Cell. Biol., 68, 450-456.

ORR, H. A. AND IRVING, S. 1997. The genetics of adaptation: the genetic basis of resistance to wasp parasitism in Drosophila melanogaster. Evolution, 51, 1877-1885.

OSBORNE, K. A., ROBICHON, A., BURGESS, E., BUTLAND, S., SHAW, R. A., COUlthard, A., PEReirA, H. S. ET AL. 1997. Natural behavior polymorphism due to a cGMP-dependent protein kinase of Drosophila. Science, 277, 834-836.

POIRIE, M., FREY, F., HITA, M., LEMEUNIER, F., PERIQUET, G. AND CARTON, Y. 2000. Drosophila resistance genes to parasitoids: 
chromosomal location and linkage analysis. Proc. R. Soc. B, 267, 1417-1421.

RIZKI, T. M. AND RIZKI, R. M. 1984. The cellular defense system of Drosophila melanogaster. In: King, R. C. and Akai, H. (eds) Insect Ultrastructure, pp. 579-603. Plenum Press, New York. SAlt, G. 1970. The Cellular Defence Reactions of Insects. Cambridge University Press, Cambridge.

SASAKI, A. AND GODFRAY, H. C. J. 1999. A model for the coevolution of resistance and virulence in coupled hostparasitoid interactions. Proc. R. Soc. B, 266, 455-463.

soKolowsKi, M. B. 1980. Foraging strategies of Drosophila melanogaster: a chromosomal analysis. Behav. Genet., 10, 291-302.

SOKOLOWSKI, M. B., BAUER, S. J., WAIPING, V., RODRIGUEZ, L., WONG, J. L. AND KENT, C. 1986. Ecological genetics and behaviour of Drosophila melanogaster larvae in nature. Anim. Behav., 34, 403-408.

STRAND, M. R. AND PECH, L. L. 1995. Immunological basis for compatibility in parasitoid-host relationships. Ann. Rev. Entomol., 40, 31-56.

WAJNBERG, E., PRÉvost, G. AND BoulÉTreAu, M. 1985. Genetic and epigenetic variation in Drosophila larvae suitability to a hymenopterous endoparasitoid. Entomophaga, 30, 187-191 WAJNBERG, E., BOULÉTREAU, M., PRÉVOST, G. AND FOUILLET, P. 1990. Developmental relationships between Drosophila larvae and their endoparasitoid Leptopilina (Hymenoptera: Cynipidae) as affected by crowding. Arch. Insect Biochem. Physiol., 13, 239-245. 